\title{
Evaluation of the Long-term Effect of Ethambutol Usage on the Retinal Nerve Fiber Layer Using Spectral-Domain Optical Coherence Tomography
}

\author{
(D) Gul Varan, ${ }^{1}$ (D) Leyla Hazar, ${ }^{2}$ (D) Ahmet Sahin ${ }^{3}$ \\ 1'Department of Ophthalmology, Corlu State Hospital, Tekirdag, Turkey \\ ${ }^{2}$ Department of Ophthalmology, Kiziltepe State Hospital, Kiziltepe, Mardin \\ ${ }^{3}$ Department of Ophthalmology, Kepez State Hospital, Kepez, Antalya
}

\begin{abstract}
Objectives: The aim of this study was to use spectral-domain optical coherence tomography (SD-OCT) to evaluate the long-term effect of ethambutol on the optic nerve fiber layer.

Methods: Twenty eyes of 10 patients who had been treated with ethambutol for tuberculosis were retrospectively evaluated. Retinal nerve fiber layer (RNFL) analyses performed initially and about 2 years after the end of treatment were included in the study. Data of visual acuity, fundus examination, other diseases, and drug use history of all of the participants were evaluated. Color vision was tested with the Ishihara Color Test. Superior, inferior, nasal, and temporal quadrant RNFL parameters were evaluated using SD-OCT.

Results: The mean age of the 10 patients was $38.77 \pm 14.86$ years. There were 6 males and 4 females in the group. The mean length of follow-up was $4.44 \pm 1.94$ months (range: 2-8 months). The mean RNFL result of the analysis performed 2 years after discontinuation of ethambutol treatment was superior RNFL: $122.45 \pm 18.64 \mu$, inferior RNFL: $131.40 \pm 13.31 \mu$, temporal RNFL: $71.60 \pm 8.53 \mu$, and nasal RNFL: $81.70 \pm 9.73 \mu$. There was significant thinning in the temporal quadrant $(p<0.05)$. Conclusion: Long-term use of ethambutol caused thinning in the RNFL. SD-OCT is useful in the follow-up of these patients. Keywords: Ethambutol, optical coherence tomography, optic neuropathy.
\end{abstract}

\section{Introduction}

Tuberculosis (TB) remains an important health problem worldwide and the subject of ongoing research. The TB incidence rate in Turkey in 2013 was $20 / 100.000$ with a mortality rate of 0.42 per $100.000(\mathrm{I})$. Ethambutol is an important first-line drug used in the treatment of TB; however, the drug has an important potential side effect on the eye: the incidence of ethambutol-induced optic neuropathy has been reported to be I-5\% (2). In the subacute clinical picture, redgreen vision is impaired and is accompanied by painless vision loss and cecocentral scotoma. A fundus examination is typ- ically normal. The clinical picture generally improves within weeks and months after discontinuation of drug treatment, but cases with persistent vision loss despite drug discontinuation have also been reported (3-6). Therefore, it is important to monitor optic nerve damage in ethambutol-induced optic neuropathies.

Spectral-domain optical coherence tomography (SD$\mathrm{OCT}$ ) is a commonly used imaging technique that quantitatively evaluates the retinal nerve fiber layer (RNFL) and the macula. The aim of this study was to analyze the longterm effect of ethambutol on the RNFL using SD-OCT using quantitative measurement values. 


\section{Methods}

The records of patients treated at Çorlu State Hospital Hospital for TB between November 2015 and March 2019 were evaluated retrospectively in terms of ethambutol toxicity. This study was conducted according to the principles of the Helsinki Declaration regarding studies involving human subjects. Approval was obtained from the ethics committee of Tekirdağ Namık Kemal University (protocol no: 2019.106.07.02).

The study included 20 eyes of 10 patients who had used ethambutol as an antituberculosis treatment and a control group of 20 eyes of 10 healthy individuals who presented at the outpatient clinic for routine eye examinations.

Patients with eye diseases such as glaucoma, age-related macular degeneration, which can affect the retinal nerve fiber thickness and macula, and a history of the use of other drugs, as well as systemic diseases like diabetes and hypertension were not included in the study. Pathological findings in the eye during drug use were also assessed. The patient dosage of ethambutol was $15 \mathrm{mg} / \mathrm{kg} /$ day at the start of treatment for a period of 2-8 months and the patients were evaluated again about 2 years after the end of treatment using SD-OCT (3D OCT-2000 Spectral Domain OCT; Topcon Medical Systems, Oakland, NJ, USA). All of the OCT images were obtained by a single experienced technician. The RNFL thickness analysis of the treatment group 2 years after discontinuation of ethambutol was compared with the RNFL thickness parameters measured in healthy individuals of the same age and gender. The mean superior, inferior, nasal, and temporal quadrant RNFL thickness measurements were included in the study. The Ishihara Color Test was used to assess the color vision of all of the participants.

\section{Statistical Analysis}

PASW Statistics for Windows, Version 18.0 software (SPSS Inc., Chicago, IL, USA) was used for the analysis of RNFL thickness. A Wilcoxon signed-rank test was used for patients who had used ethambutol, and a Mann-Whitney $U$ test was used for the comparison with healthy individuals. A $P$ value $<0.05$ was considered significant.

\section{Results}

The mean age in Group I (treatment group) and Group 2 (control group) was $38.77 \pm 14.86$ years and $42.4 \pm 17.94$ years, respectively $(p=0.505)$. The male/female ratio was $6 / 4$ and $7 / 3$ in Group I and Group 2, respectively ( $p=0.507)$. There was no significant difference between the groups in terms of age or gender. The mean length of follow-up in the patient group was $4.44 \pm 1.94$ months (range: $2-8$ months). The mean Snellen chart visual acuity was measured as 1.0; no pathology was found in other ophthalmologic examination findings. The Ishihara color vision test findings were normal in all of the study participants. The mean RNFL thickness parameters of the ethambutol users at the beginning of treatment and the second year after treatment are shown in Table I. The mean RNFL thickness parameters of the healthy adults are shown in Table 2. The comparison of RNFL thickness

Table I. RNFL thickness values recorded at the start of ethambutol treatment (I ${ }^{\text {st }}$ measurement) and 2 years after cessation of treatment $\left(2^{\text {nd }}\right.$ measurement $)$

\begin{tabular}{lccc} 
& $\begin{array}{c}\mathbf{~}^{\text {st }} \text { measurement } \\
(\boldsymbol{\mu m})\end{array}$ & $\begin{array}{c}\mathbf{2}^{\text {nd }} \text { measurement }(\boldsymbol{\mu m}) \\
(\text { Mean } \pm \text { SD) }\end{array}$ & * $\mathbf{p}$ \\
\hline Superior RNFL & $122.40 \pm 17.82$ & $122.45 \pm 18.64$ & 0.491 \\
Inferior RNFL & $131.60 \pm 11.62$ & $131.40 \pm 13.31$ & 0.837 \\
Nasal RNFL & $81.20 \pm 12.50$ & $81.70 \pm 9.73$ & 0.881 \\
Temporal RNFL & $76.20 \pm 10.68$ & $71.60 \pm 8.53$ & 0.016
\end{tabular}

*Wilcoxon test; RNFL: Retinal nerve fiber layer.

Table 2. RNFL thickness values of the control group and the measurements taken 2 years after termination of ethambutol treatment

\begin{tabular}{|c|c|c|c|}
\hline & $\begin{array}{c}\text { Control }(\mu \mathrm{m}) \\
\text { (Mean士SD) }\end{array}$ & $\begin{array}{c}2^{\text {nd }} \text { year }(\mu \mathrm{m}) \\
(\text { Mean } \pm S D)\end{array}$ & $* \mathbf{p}$ \\
\hline Superior RNFL & $125.00 \pm 13.77$ & $122.45 \pm 18.64$ & 0.845 \\
\hline Inferior RNFL & $|28.27 \pm| 4.48$ & $|3| .40 \pm|3.3|$ & 0.437 \\
\hline Nasal RNFL & $85.77 \pm 16.34$ & $81.70 \pm 9.73$ & 0.338 \\
\hline Temporal RNFL & $72.97 \pm 9.09$ & $71.60 \pm 8.53$ & 0.677 \\
\hline
\end{tabular}

*Mann-Whitney U test; RNFL: Retinal nerve fiber layer. 
analysis revealed a significant thinning in the temporal quadrant in the second year in patients treated with ethambutol. In comparison with the healthy individuals, the thickness of the superior, nasal, and temporal RNFL quadrants had decreased, but did not reach the level of statistical significance.

\section{Discussion}

The first side effect of ethambutol, which has been used as a first-line drug in the treatment of tuberculosis since 196I, was reported by Carr and Henkind in 1962 (7). Leibold reported in 1966 that eye toxicity was significantly reduced when ethambutol was given for a short time and with a dose of $<25 \mathrm{mg} / \mathrm{kg} /$ day (8). Recent studies have shown that the incidence of developing optic neuropathy falls below $1 \%$ when the daily dose is $15 \mathrm{mg} / \mathrm{kg} /$ day (2). Use of ethambutol has been shown to affect the papillomacular fiber bundles in cases of central neuropathy, leading to central scotoma and a decrease in visual acuity and green vision (5). A fundus examination in the early stage of treatment is usually normal. Optic nerve findings usually appear months after visual acuity is affected (9). Therefore, it is very difficult to evaluate the optic nerve in the first stage. SD-OCT allows for the precise measurement of the optic nerve and retina layers. This provides a differential diagnosis. In recent years, OCT has been widely used for diagnosis and follow-up in clinical and subclinical neuro-ophthalmological diseases ( 10$)$.

The selective toxicity of ethambutol to the optic nerve is particularly focused on mitochondrial dysfunction (5-9). Histopathological findings of ethambutol-induced optic neuropathies have been found to be similar to mitochondrial hereditary optic neuropathies (1I-13). Ethambutol, which is a metal ion chelator, reduces ATPase activity by decreasing copper and iron levels in cytochrome-c in mitochondria, and a low adenosine triphosphate level disrupts axoplasmic transport. The neurons in the papillomacular bundle are more sensitive to low energy because they are low-caliber axons and have a thin myelin sheath (I I-I3).

In the first stage, swelling occurs in neuron axons in the papillomacular band (5, II-13). The papillomacular band corresponds to the temporal quadrant in RNFL analysis.

In our study, a statistically significant thinning was found in the comparison of the temporal quadrant of RNFL values recorded at the beginning of ethambutol treatment and 2 years after treatment. In a previous study, Taffner et al. (14) evaluated the RNFL analysis of 30 patients before and after ethambutol treatment at 2-month intervals. They found that 2-month standard treatment and 9-12-month extended treatment led to a significant reduction in the RNFL, and prolonged treatment caused further thinning.

Chai et al. (4) compared the RNFL thickness measured at the initial exam and 3 months after treatment termination in
8 patients with ethambutol-induced optic neuropathy. They found significant thinning in the superior and nasal quadrants and particularly in the temporal quadrant. We found consistent results with a similar group, including the number of participants.

Zoulaman et al. (5) compared 3 patients with ethambutol-induced optic neuropathy whose visual acuity had been severely affected and whose drug treatment had been long terminated (range: 6 months- 5 years) with the RNFL analysis of healthy individuals. They reported that they found a RNFL thickness reduction in all quadrants, and especially the temporal quadrant.

In our study, when we compared patients who had terminated ethambutol treatment 2 years earlier with healthy individuals of the same age and gender, we found thinning in the temporal, superior, and nasal quadrants. However, a weakness of our study was not to evaluate the relationship between the RNFL thinning and the visual field defect.

Ungsoo et al. (15) compared the measurements of 10 patients who presented within 6 months after the development of ethambutol-induced optic neuropathy symptoms and 54 healthy eyes. They found an increase in the RNFL thickness in the temporal quadrant, although it was not statistically significant. The authors reported that this increase in thickness may have been due to axonal swelling in the papillomacular band in the initial stage of optic neuropathy. A strength of our study was the comparison of the analysis of the second year after cessation of ethambutol with the initial treatment.

In conclusion, it has been shown that ethambutol treatment may have a long-term effect on the RNFL after discontinuation. SD-OCT is very useful for obtaining objective, quantitative measurements to monitor the effects of ethambutol on the optic nerve. Nonetheless, more studies are needed to more clearly evaluate the long-term effect of ethambutol treatment on the optic nerve fiber layer.

\section{Disclosures}

Ethics Committee Approval: Tekirdağ Namık Kemal University protocol no: 2019.106.07.02.

Peer-review: Externally peer-reviewed.

Conflict of Interest: None declared.

Authorship Contributions: Involved in design and conduct of the study (GV, LH, AS); preparation and review of the study (GV, $\mathrm{LH}, \mathrm{AS}$ ); data collection (GV, LH, AS); and statistical analysis (GV, LH, AS).

\section{References}

I. Etiz P, Altınsu AT. A Retrospective Analysis of 232 Newly Diagnosed Cases with Pulmonary tuberculosis, Türk Mikrobiyol Cem Derg 2018;48: I 17-24. [CrossRef]

2. Sivakumaran P, Harrison AC, Marschner J, Martin P. Ocular 
toxicity from ethambutol: a review of four cases and recommended precautions. N Z Med J 1998; I I I:428-30.

3. Kumar A, Sandramouli S, Verma L, Tewari HK, Khosla PK. Ocular ethambutol toxicity: is it reversible?. J Clin Neuroophthalmol 1993;13:15-7.

4. Chai SJ, Foroozan R. Decreased retinal nerve fibre layer thickness detected by optical coherence tomography in patients with ethambutol-induced optic neuropathy. $\mathrm{Br} J$ Ophthalmol 2007;91:895-7. [CrossRef]

5. Zoumalan $\mathrm{Cl}$, Agarwal M, Sadun AA. Optical coherence tomography can measure axonal loss in patients with ethambutol-induced optic neuropathy. Graefes Arch Clin Exp Ophthalmol 2005;243:410-6. [CrossRef]

6. Bıçakçı Ş, Özeren A, Yerdelen D, Sarıca Y. Optic Neuropathy Due To Ethambutol Intake: A Case Report. Türkiye Klinikleri J Med Sci 2005;25:460-2.

7. CARR RE, HENKIND P. Ocular manifestations of ethambutol, Toxic amblyopia after administration of an experimental antituberculous drug. Arch Ophthalmol 1962;67:566-7I. [CrossRef]
8. Leibold JE. The ocular toxicity of ethambutol and its relation to döşe. Ann NY Acad Sci 1966;135:904-9. [CrossRef]

9. Chan RY, Kwok AK. Ocular toxicity of ethambutol. Hong Kong Med J 2006; 12:56-60.

10. Mudun A. Optical Coherence Tomography in Neuro-Ophthalmological Diseases. ACU Sağlık Bil Derg 201 I;2:5-9.

I I. Sadun A. Acquired mitochondrial impairment as $s$ cause of optic nerve disease. Trans Am Ophthalmol Soc 1998:88I-923.

12. Carelli V, Ross-Cisneros FN, Sadun AA. Optic nerve degeneration and mitochondrial dysfunction: genetic and acquired optic neuropathies. Neurochem Int 2002;40:573-84. [CrossRef]

13. Sadun AA. Mitochondrial optic neuropathies. J Neurol Neurosurg Psychiatry 2002;72:423-5.

14. Pavan Taffner BM, Mattos FB, Cunha MCD, Saraiva FP. The use of optical coherence tomography for the detection of ocular toxicity by ethambutol. PLoS One 20 I8; I3:e0204655. [CrossRef]

I5. Kim U, Hwang JM. Early stage ethambutol optic neuropathy: retinal nerve fiber layer and optical coherence tomography. Eur J Ophthalmol 2009;19:466-9. [CrossRef] 